\title{
Study of the triplet and pair structure of strong electrolytes modeled via truncated Coulomb interactions
}

\author{
S. Jorge ${ }^{\text {a) }}$ \\ Instituto de Química Física Rocasolano, CSIC, Serrano 119, E-28006 Madrid, Spain and Departamento de \\ Química Física I, Universidad Complutense, E-28040 Madrid, Spain \\ E. Lomba \\ Instituto de Química Física Rocasolano, CSIC, Serrano 119, E-28006 Madrid, Spain \\ J. L. F. Abascal \\ Departamento de Química Física I, Universidad Complutense, E-28040 Madrid, Spain
}

(Received 29 April 2002; accepted 30 May 2002)

\begin{abstract}
The structure of 3:3 and 1:3 electrolyte solutions at various concentrations and several cation/anion size ratios has been analyzed in terms of triplet and pair correlation functions, by means of simulation and a triplet integral equation theory derived from the inhomogeneous Ornstein-Zernike equation. The interaction model consists of a truncated and shifted Coulomb plus the RamanathanFriedman repulsive core. Concentration and size and charge asymmetry are found to induce changes in the triplet structure beyond those predicted by the simple superposition approximation, which are, however, correctly reproduced by the triplet integral equation. (C) 2002 American Institute of Physics. [DOI: 10.1063/1.1495400]
\end{abstract}

\section{INTRODUCTION}

The theoretical study of electrolytes, and in particular association phenomena, has developed increasing interest over the last decades. The simplest approximations at hand view the electrolyte within the McMillan-Mayer ${ }^{1}$ picture in which the effect of solvent molecules is averaged out and incorporated into the ion-ion pair potential by means of the dielectric constant of the medium. Despite the fact that solvation effects are explicitly neglected in this approach, in many cases of interest it is well suited to describe the ionion structural properties and thermodynamics (activity coefficients, osmotic pressure, and related quantities).

Once we restrict ourselves to the McMillan-Mayer level of description, an electrolyte reduces to a mixture of charged particles that, in the simplest case, are spherical. In this case the system is amenable to be treated in the well-established framework of integral equation theories for mixtures of simple fluids. Among these, a successful approach for charged systems is the hypernetted chain equation (HNC), which has proved to be remarkably accurate from dilute to moderately concentrated electrolyte solutions. ${ }^{2}$ The success of the HNC for these low density systems rests upon its correct treatment of ionic pairing, being an exact approximation at the second virial coefficient level. For high densities, i.e., in the molten salt regime, the HNC can be improved using a hard sphere reference bridge function ${ }^{3}$ with relatively good results. ${ }^{4}$ Nonetheless, in the strong electrolyte regime the HNC approximation exhibits important deficiencies that are due to the increasing role of clustering as the ionic strength is augmented. ${ }^{5}$ Some improvement was achieved reformulating the functional form of the bridge function with

${ }^{a)}$ Electronic mail: sonia@iqfr.csic.es the aid of computer simulation, as was done by Duh and Haymet $^{6}$ in the INV approximation. Along the same lines, Bresme et $_{\text {al. }}{ }^{7}$ developed the INV-C integral equation. Both approaches were fairly successful in cases where the HNC fails to reproduce the electrolyte structure, but the semiphenomenological nature of these treatments has prevented further improvements so far.

On the other hand, approximations based on Wertheim's association theory ${ }^{8}$ like the multidensity integral equation ${ }^{9}$ represent a promising alternative for an explicit account of clustering effects in electrolytes.

Our approach, however, aims at exploring these clustering effects from the standpoint of three particle correlations, which implies that our departure level is already beyond simple pairing. We will thus use the inhomogeneous Ornstein-Zernike (IOZ) approach proposed by Attard, ${ }^{10}$ which has recently been extended by us to describe binary mixtures. ${ }^{11}$ In this approach, following Percus source particle method, a given particle is considered as the source of an external potential and hence an IOZ can be constructed to determine a series of inhomogeneous correlation functions which are trivially related to the homogeneous correlation functions of higher order. In addition, the inhomogeneous density is connected with the inhomogeneous pair distribution function by means of a Triezenberg-ZwanzigWertheim-Lovett-Mou-Buff $\quad$ (TZWLMB $)^{12,13}$ integral equation. As cited before, previous work in electrolyte systems suggests that the HNC approximation seems to be the appropriate closure for the IOZ. The set of equations thus constructed - usually denoted by $\mathrm{HNC} 3$ - has shown to remarkably improve the performance of the plain HNC for simple systems, ${ }^{10}$ fluids with inherent icosahedral order, ${ }^{14}$ and mixtures. ${ }^{11}$ Obviously, the approximation at the three particle level contains information on the local fluid geom- 
TABLE I. Simulation conditions and thermodynamics states considered in this work. The ionic strength is defined by $I=\frac{1}{2} \Sigma_{\alpha} c_{\alpha} q_{\alpha}^{2}$ and the packing fraction is $\eta=\pi\left(\rho_{-} \sigma_{--}^{3}+\rho_{+} \sigma_{++}^{3}\right) / 6$.

\begin{tabular}{|c|c|c|c|c|c|c|c|c|}
\hline Electrolyte & $\sigma_{+} / \sigma_{-}$ & $c(\mathrm{M})$ & $\epsilon$ & $\eta$ & $I(\mathrm{M})$ & $r_{\mathrm{ts}}^{*}$ & $N_{\text {part }}$ & $N_{\mathrm{st}} \times 10^{-3}$ \\
\hline \multirow{8}{*}{$3: 3$} & \multirow{5}{*}{1.0} & 0.89 & 78.36 & 0.0129 & 80.0 & 6.5 & 500 & 1350 \\
\hline & & 7.78 & 78.36 & 0.1127 & 70.0 & 6.5 & 864 & 300 \\
\hline & & 8.89 & 78.36 & 0.1288 & 80.0 & 6.5 & 1372 & 300 \\
\hline & & 7.78 & 78.36 & 0.1127 & 70.0 & 7.5 & 1372 & 300 \\
\hline & & 8.89 & 78.36 & 0.1288 & 80.0 & 7.5 & 1372 & 300 \\
\hline & \multirow[t]{2}{*}{2.0} & 0.89 & 78.36 & 0.0580 & 8.0 & 18.5 & 1372 & 300 \\
\hline & & 1.67 & 78.36 & 0.1089 & 15.0 & 16.5 & 2048 & 150 \\
\hline & 3.0 & 0.89 & 78.36 & 0.1806 & 8.0 & 18.5 & 1372 & 150 \\
\hline \multirow[t]{3}{*}{$1: 3$} & \multirow[t]{2}{*}{1.707} & 1.33 & 78.36 & 0.0768 & 8.0 & 12.5 & 1728 & 150 \\
\hline & & 2.50 & 78.36 & 0.1444 & 15.0 & 12.5 & 2744 & 150 \\
\hline & 3.0 & 0.89 & 78.36 & 0.1934 & 5.35 & 18.5 & 2744 & 150 \\
\hline $1: 3$ & 1.707 & 1.33 & 36.40 & 0.0768 & 8.0 & 12.5 & 1728 & 150 \\
\hline
\end{tabular}

etry which is unavailable to pair approximations. Consequently, it is our intention here to explore the capabilities of the HNC3 approach to describe the behavior of electrolytes up to rather high ionic strengths, both in the fully symmetric
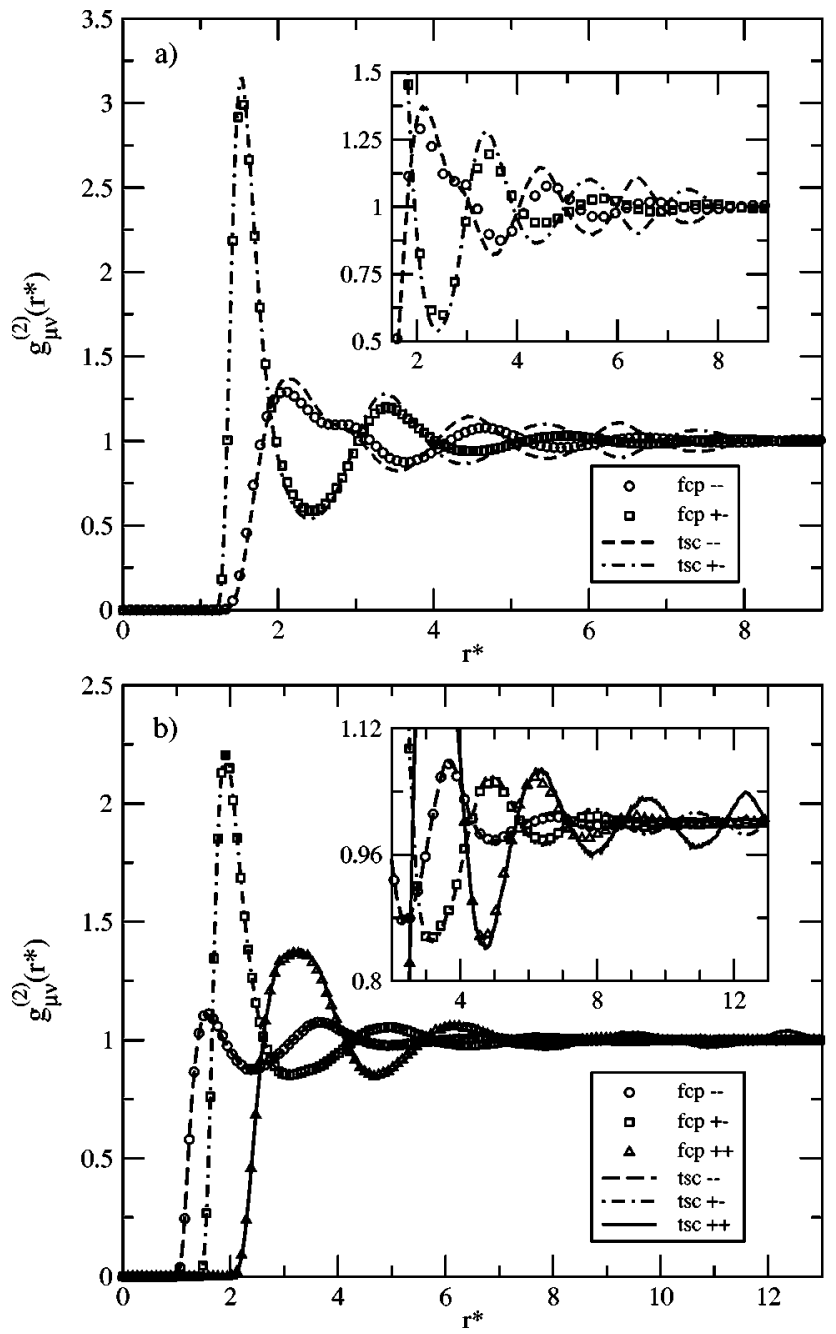

FIG. 1. Pair distribution function results from MD for a truncated and shifted Coulomb interaction (tsc, lines) and full Coulomb potential ( $\mathrm{fcp}$, symbols) in: (a) a 3:3 electrolyte with $\lambda=1.0$ and $r_{\mathrm{ts}}^{*}=6.5$ at $c=7.78 \mathrm{M}$; (b) a $1: 3$ electrolyte with $\lambda=1.707$ and $c=2.5 \mathrm{M}$. case and with size and charge asymmetry. For comparison purposes, in some cases the approach proposed by Barrat, Hansen, and Pastore ${ }^{15}$ and extended to mixtures in Ref. 16 has also been utilized.

The long range of the Coulomb interaction poses addi-

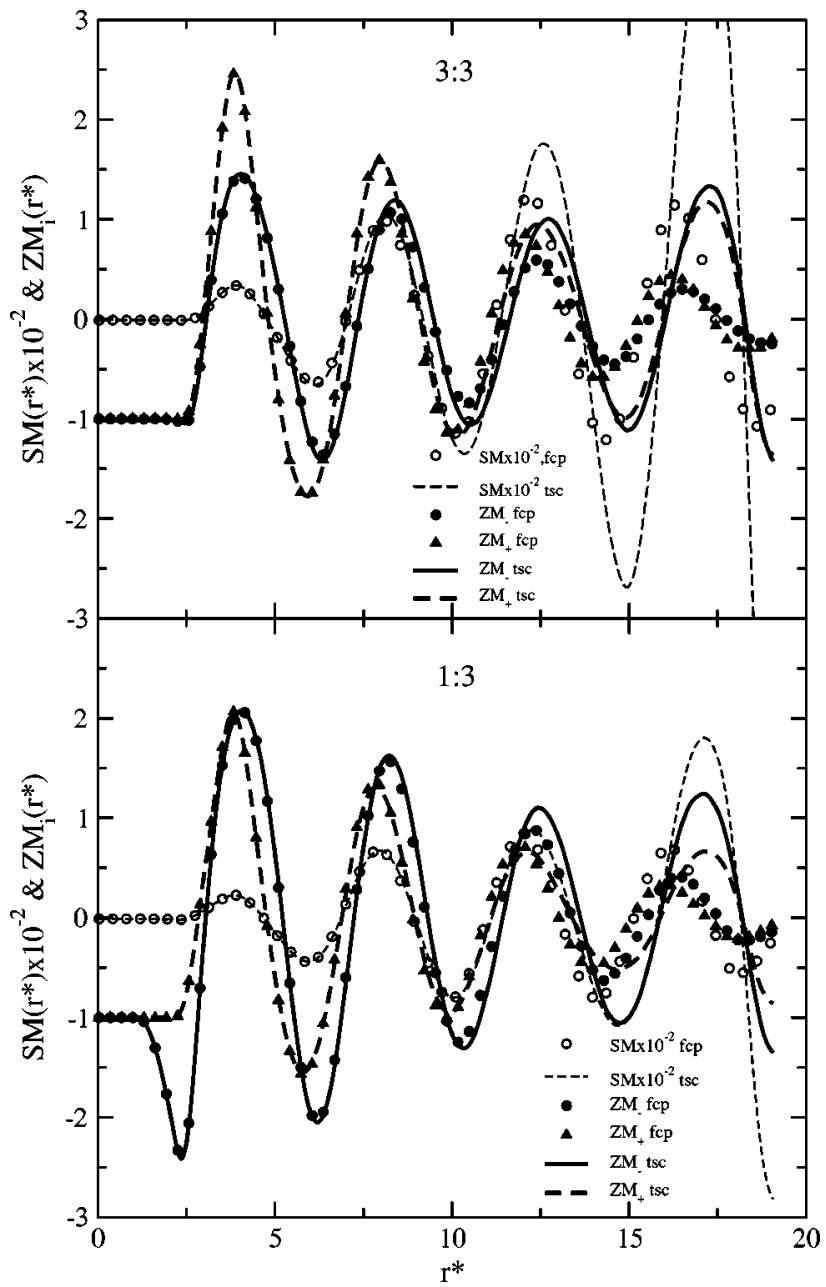

FIG. 2. Stillinger-Lovett zero and second moments for electrolyte solutions with $\lambda=3$ at $c=0.89$ M. Symbols: fcp results. Lines: tsc results. Upper graph. 3:3 electrolyte solution. Lower graph. 1:3 electrolyte solution. The notation is explained in the legend box. 

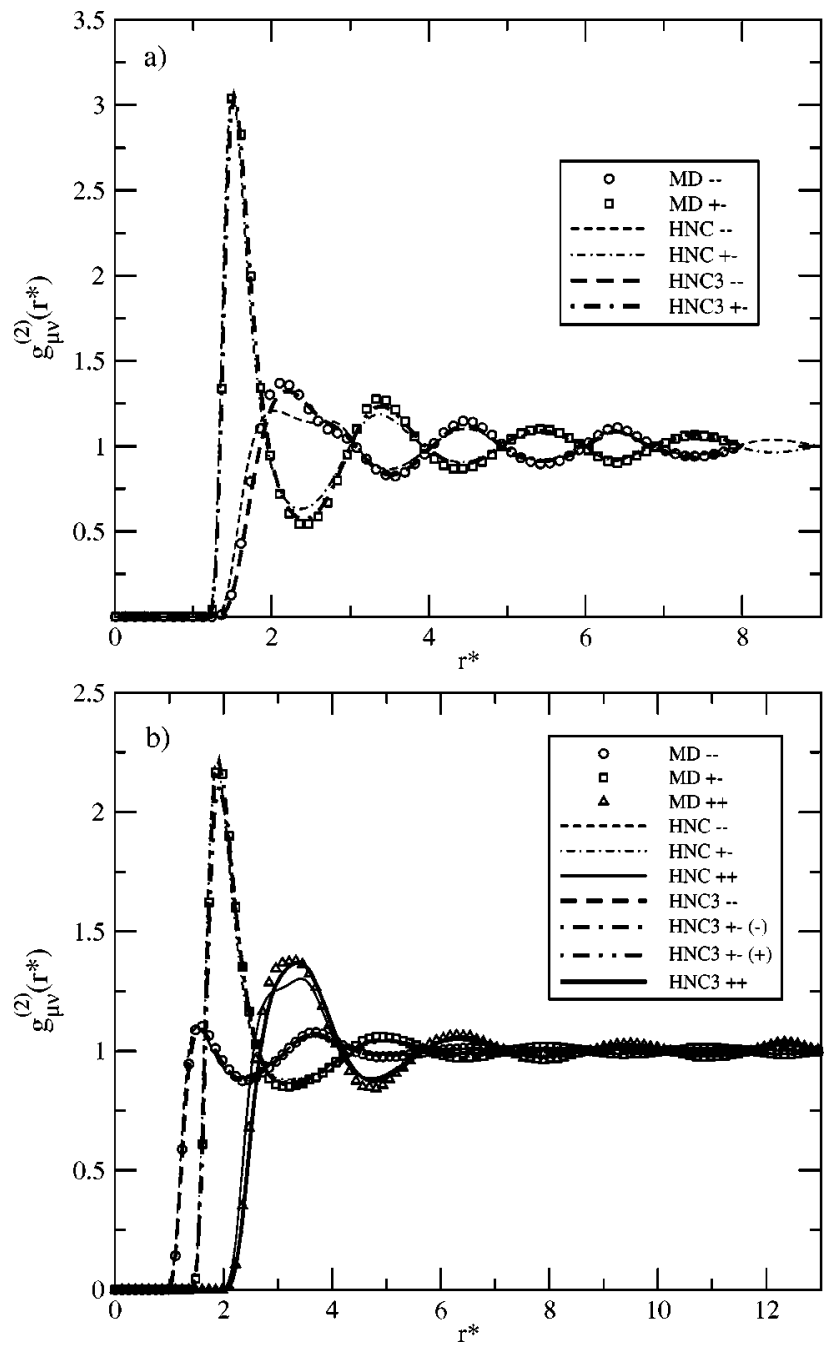

FIG. 3. Theoretical vs MD pair distribution functions for the truncated and shifted potential of systems (a) and (b) in Fig. 1.

tional difficulties for the solution of the HNC3 equation, by itself quite demanding from the computational standpoint. In principle one could rewrite the HNC3 equations in a renormalized form following Allnat's procedure for the pair HNC equation. ${ }^{17}$ However, the fact that the HNC3 must be solved in real space does not allow for a straightforward extension of the renormalization approach, best suited for a Fourier space formalism. One will have then to cope with the integral equation resorting to longer integration ranges than those considered in Lennard-Jones mixtures ${ }^{11}$ but subject to computer storage limitations. Consequently we have opted here for a truncated and shifted Coulomb potential to model the electrolyte. This model, in which the core repulsions are represented by a Ramanathan-Friedman interaction, ${ }^{18}$ is known to yield a reasonable picture of electrolyte systems in a wide variety of physical conditions. ${ }^{19}$ This is especially true for the low density-high ionic strength regime in which most of the ions are associated into neutral clusters and hence the intercluster interaction is of multipolar character, i.e., it decays much more rapidly than the pure unshielded Coulomb potential. In particular, we are mostly interested in the short range structure which results from clustering effects essentially due to the short and medium range regions of the
Coulomb interaction. We will see that in the cases considered herein the replacement of the pure Coulomb by its truncated and shifted counterpart introduces only minor changes in the electrolyte structure.

The rest of the paper can be sketched as follows. In Sec. II we present the details of the interaction model. Section III is devoted to a brief summary of the HNC3 and the BHP theory and their numerical implementation. Finally, our most significant results are commented upon in Sec. IV.

\section{TRUNCATED-SHIFTED COULOMB POTENTIAL}

As mentioned before, the model potential used here has been widely utilized in other works ${ }^{18,20-22}$ to model various electrolyte solutions. The interaction consists of a repulsive short-range core and a Coulomb tail, which is truncated and shifted according to

$$
V_{\mu \nu}^{\mathrm{tsc}}(r)=\left\{\begin{array}{cc}
u_{\mu \nu}^{\mathrm{RF}}(r)+u_{\mu \nu}^{c}(r)-P_{\mu \nu}\left(r_{\mathrm{ts}}\right), & 0<r<r_{t} \\
u_{\mu \nu}^{\mathrm{RF}}(r)+P_{\mu \nu}(r)-P_{\mu \nu}\left(r_{\mathrm{ts}}\right), & r_{t}<r<r_{t s} \\
0, & r>r_{\mathrm{ts}}
\end{array}\right\},
$$

where the short range repulsive term is the RamanathanFriedman potential, $u_{\mu \nu}^{\mathrm{RF}}(r)$,

$$
u_{\mu \nu}^{\mathrm{RF}}(r)=\frac{5377.75\left|q_{\mu} q_{\nu}\right|}{\left(r_{\mu}+r_{\nu}\right)}\left(\frac{r_{\mu}+r_{\nu}}{r}\right)^{9}
$$

and $u_{\mu \nu}^{c}(r)$ represents the Coulomb term,

$$
u_{\mu \nu}^{c}(r)=\frac{q_{\mu} q_{\nu} e^{2}}{\epsilon r}
$$

with $q_{\mu}$ the particle charge (in electron charge units), $\epsilon$ the dielectric constant, $e$ the electron charge, and $r_{\mu}$ and $r_{\nu}$ represent the radii of ions $\mu$ and $\nu$. Finally, in Eq. (1) $r_{t}$ is the cutoff radius of the full potential, and $r_{\mathrm{ts}}$ is the cutoff distance of the Coulomb potential where the shifting polynomial takes over $P_{\mu \nu}(r)$. This function is defined by the following constraints:

$$
\begin{array}{ll}
P_{\mu \nu}^{\prime}\left(r_{t}\right)=u_{\mu \nu}^{c \prime}\left(r_{t}\right), & P_{\mu \nu}^{\prime \prime}\left(r_{t}\right)=u_{\mu \nu}^{c \prime \prime}\left(r_{t}\right), \\
P_{\mu \nu}\left(r_{t}\right)=u_{\mu \nu}^{c}\left(r_{t}\right), & P_{\mu \nu}^{\prime}\left(r_{\mathrm{ts}}\right)=0 .
\end{array}
$$

Here the prime and double prime denote, respectively, the first and second derivatives. In all cases $r_{\mathrm{ts}}$ has been taken as $r_{\mathrm{ts}}=r_{t}+r_{\alpha}$, where $r_{\alpha}$ is the radius of the smallest particle. In our models the anions are the smallest particles.

\section{A SUMMARY OF THE THEORY}

The IOZ equation when formulated in the context of the source particle method of Percus constitutes the foundation of the HNC3 approximation. ${ }^{10}$ As mentioned before the inhomogeneity of the system stems from a particle located at the origin of a given triplet, which breaks the translational invariance of the density. The problem then reduces to solving the IOZ equation complemented with a closure relation and a given density profile. For electrolyte solutions we can resort to the multicomponent formulation of this approach. ${ }^{11}$ 

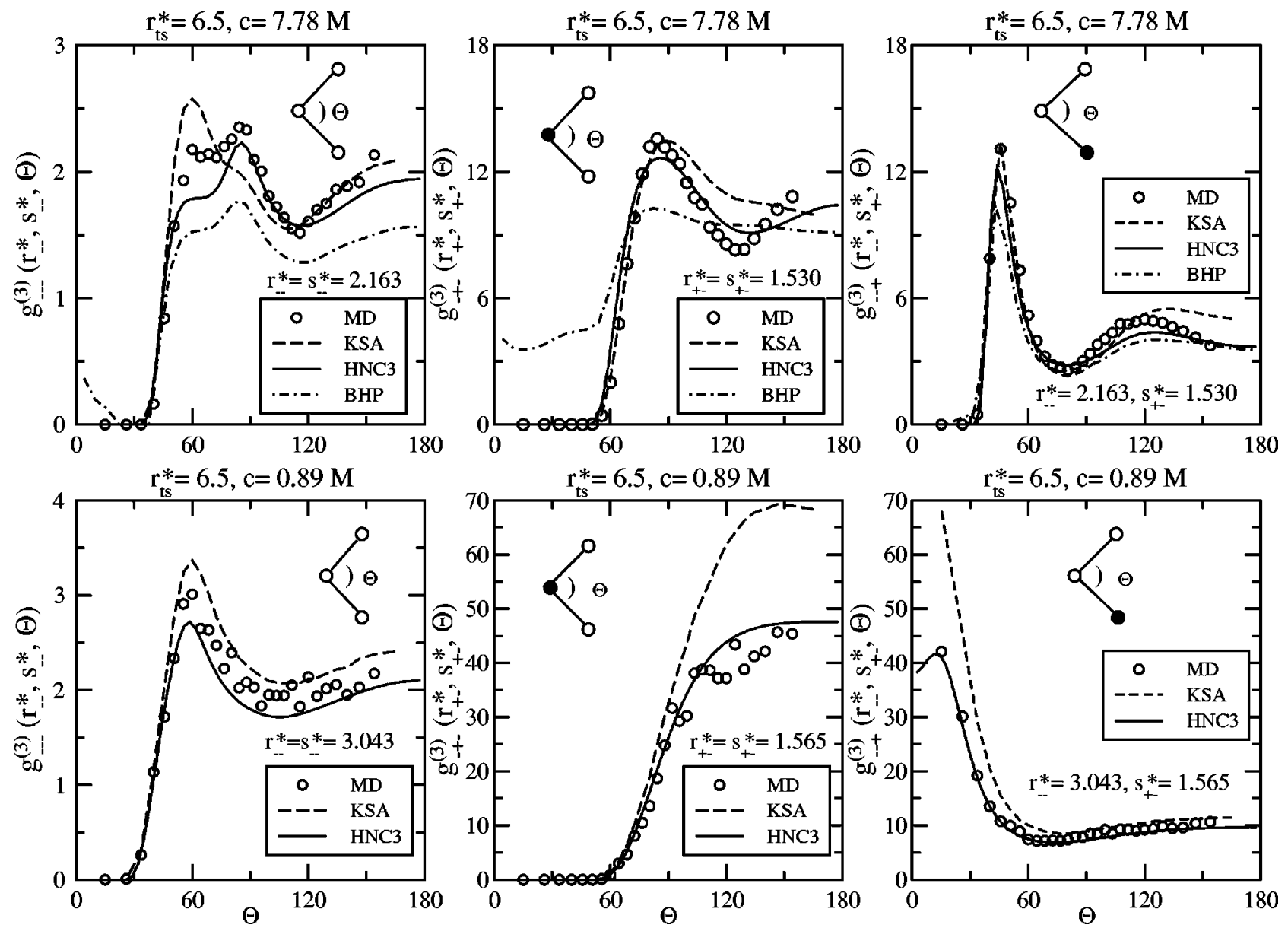

FIG. 4. Triplet distribution function for a 3:3 electrolyte solution with $\lambda=1.0$. The three upper graphs are at $c=7.78 \mathrm{M}$ and the three lower graphs are at $c=0.89 \mathrm{M}$

For a mixture of $n$ components $(\mu, \nu, \ldots)$ the IOZ equation which relates the pair total correlation function $h_{\mu \nu \gamma}^{(2)}\left(\boldsymbol{r}_{1}, \boldsymbol{r}_{2}\right)$ to the pair direct correlation function $c_{\mu \nu \underline{\gamma}}^{(2)-}\left(\boldsymbol{r}_{1}, \boldsymbol{r}_{2}\right)$ reads

$$
\begin{aligned}
h_{\mu \nu \underline{\gamma}}^{(2)}\left(r_{1}, r_{2}, \theta_{12}\right)= & c_{\mu \nu \underline{\gamma}}^{(2)}\left(r_{1}, r_{2}, \theta_{12}\right) \\
& +\sum_{\lambda=1}^{n} \int d r_{4} \rho_{\lambda \underline{\gamma}}\left(r_{4}\right) c_{\mu \lambda \underline{\gamma}}^{(2)}\left(r_{1}, r_{4}, \theta_{14}\right) \\
& \times h_{\lambda \nu \underline{\gamma}}^{(2)}\left(r_{4}, r_{2}, \theta_{42}\right) .
\end{aligned}
$$

Particles $\mu$ and $\nu$ correspond to coordinates $\boldsymbol{r}_{1}$ and $\boldsymbol{r}_{2}$, being $\cos \theta_{i j}=\boldsymbol{r}_{i} \boldsymbol{r}_{j} / r_{i} r_{j}$. The third particle $\gamma$, located at the origin of the triplet, represents the source particle which, from here onward, will be denoted by an underlined Greek letter. As explained elsewhere, ${ }^{10,11}$ the axial symmetry of the system makes possible the Legendre transformation of the integral equation in order to simplify the calculations. In our binary electrolyte there are six equations of the type (5) arranged in two sets of three equations coupled by the density profiles.

As usual, a closure relation expresses the pair distribution functions in terms of the interaction potential, $v_{\mu \nu}\left(r_{12}\right)$. In the present instance we will focus on the HNC closure, which in the inhomogeneous case reads

$$
\begin{aligned}
g_{\mu \nu \underline{\gamma}}^{(2)}\left(r_{1}, r_{2}, \theta_{12}\right)= & \exp \left\{-\beta v_{\mu \nu}\left(r_{12}\right)+h_{\mu \nu \underline{\gamma}}^{(2)}\left(r_{1}, r_{2}, \theta_{12}\right)\right. \\
& \left.-c_{\mu \nu \underline{\gamma}}^{(2)}\left(r_{1}, r_{2}, \theta_{12}\right)\right\},
\end{aligned}
$$

where $\beta=1 /\left(K_{B} T\right)$ and $r_{12}=\left(r_{1}^{2}+r_{2}^{2}-2 r_{1} r_{2} \cos \theta_{12}\right)^{1 / 2}$.

Equations (5) and (6) have to be complemented by an equation for the density profile, $\rho_{\lambda \gamma}(r)$. Following previous works we use here the TZWLMB equation, which once Legendre transformed can be expressed as ${ }^{11}$

$$
\begin{aligned}
\rho_{\mu \underline{\gamma}}^{(2)}\left(r_{1}\right)= & \rho_{\mu} \exp \left\{-\beta v_{\mu \underline{\gamma}}\left(r_{1}\right)\right. \\
& -\int_{\infty}^{r} \frac{4 \pi}{3} \beta\left[\sum_{\lambda=1}^{n} \int_{0}^{\infty} d r_{2} r_{2}^{2} \rho_{\lambda \underline{\gamma}}\left(r_{2}\right) \hat{h}_{1}^{\mu \lambda} \underline{\gamma}\left(r_{1}, r_{2}\right)\right. \\
& \left.\left.\times \frac{d v_{\lambda \underline{\gamma}}\left(r_{2}\right)}{d r_{2}}\right] d r_{1}\right\}
\end{aligned}
$$

taking into account that $\rho_{\mu \gamma}(r)=\rho_{\mu} g_{\mu \gamma}(r)$ and $\rho\left(r_{\infty}\right)$ $\rightarrow \rho_{\text {bulk }}$. The bulk three-particle distribution function can be related to the inhomogeneous pair distribution function through

$$
g_{\mu \nu \underline{\gamma}}^{(3)}\left(r_{1}, r_{2}, \theta_{12}\right)=g_{\mu \gamma}\left(r_{1}\right) g_{\nu \gamma}\left(r_{2}\right) g_{\mu \nu \underline{\gamma}}^{(2)}\left(r_{1}, r_{2}, \theta_{12}\right) .
$$




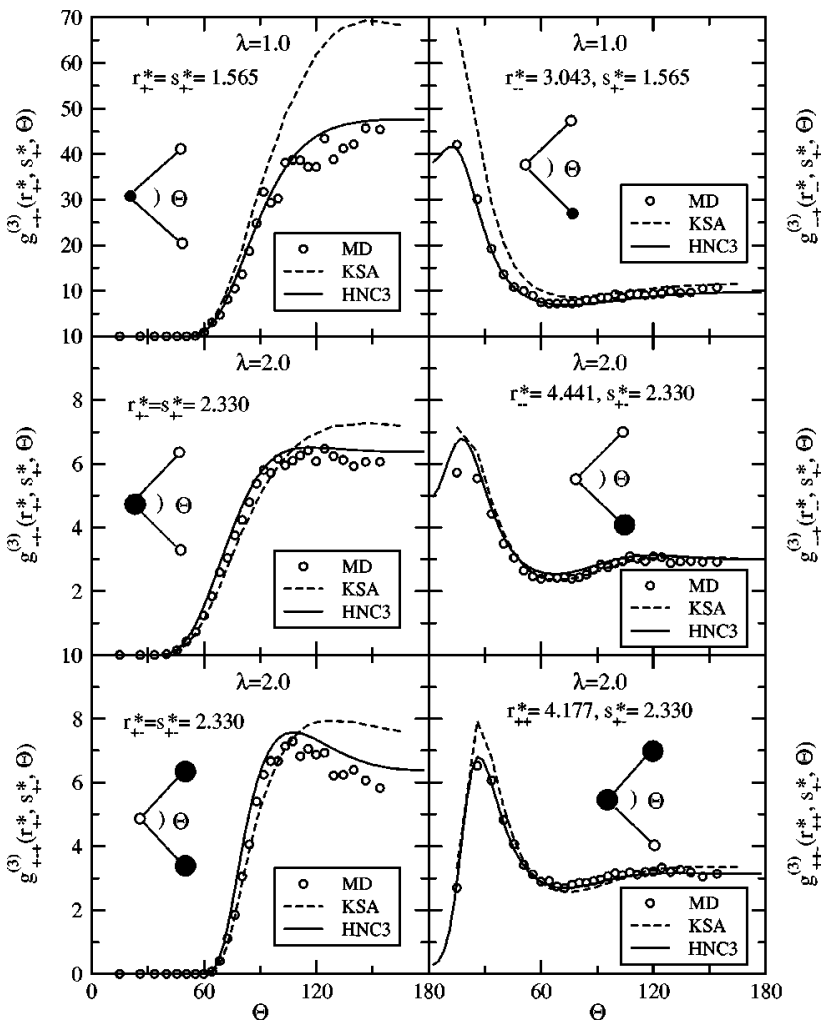

FIG. 5. Triplet distribution function for 3:3 electrolytes at $c=0.89 \mathrm{M}$ with different $\lambda$ values.

Here $g_{\mu \nu \gamma}^{(2)}\left(r_{1}, r_{2}, \theta_{12}\right)$ represents the probability of finding particles $\bar{\mu}$ and $\nu$, respectively, at distances $r_{1}$ and $r_{2}$ from the source $\gamma$.

The set of three equations is solved by means of the generalized minimal residual algorithm for nonlinear systems of equations (GMRESNL), ${ }^{23}$ whose ability to cope with large nonlinear systems of equations is well established. A complete description of this method is done elsewhere. ${ }^{11,14}$ We have discretized the inhomogeneous functions using 200-250 grid points in $r_{i}$ with a grid size $0.04 \sigma_{--}\left(\sigma_{--}\right.$

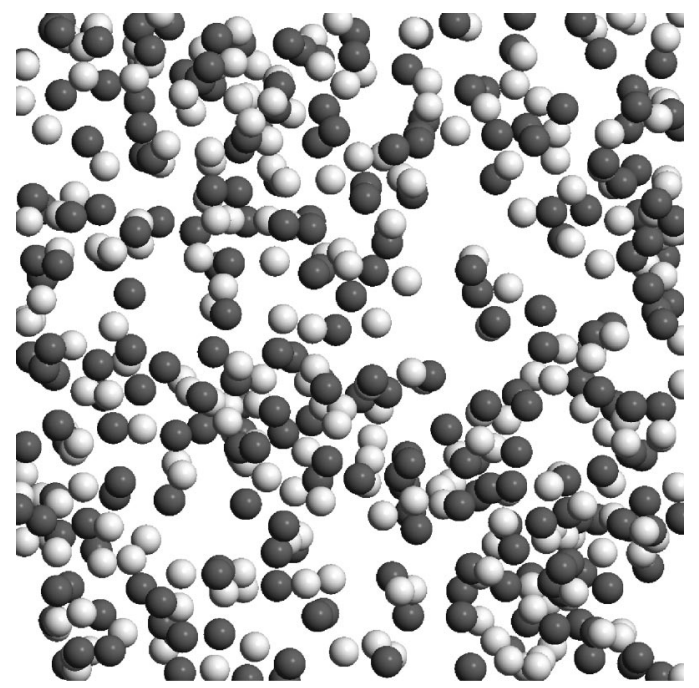

FIG. 6. Snapshot of a MD configuration of a 3:3 electrolyte with $\lambda=1.0$ at $c=0.89 \mathrm{M}$.

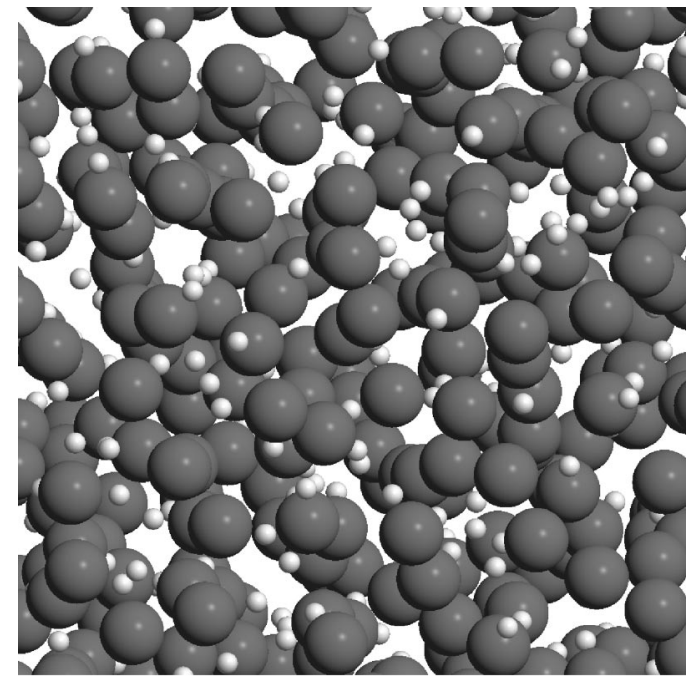

FIG. 7. Same as Fig. 6 for $\lambda=3.0$.

$\left.=2 r_{-}\right)$and the discrete Legendre transformation is carried out using 60 coefficients. Five search directions did suffice to reach convergence in the GMRESNL algorithm.

For details of the Barrat-Hansen-Pastore (BHP) theory we refer the reader to Refs. 15 and 16 . Here we just recall its most basic essentials. The BHP assumes a factorization ansatz for the triplet direct correlation function $c_{\mu \nu \xi}^{(3)}\left(\boldsymbol{r}, \boldsymbol{r}^{\prime}\right)$ of the form

$$
c_{\mu \nu \xi}^{(3)}\left(\boldsymbol{r}, \boldsymbol{r}^{\prime}\right)=t_{\mu \nu}^{\mu \nu \xi}\left(\left|\boldsymbol{r}-\boldsymbol{r}^{\prime}\right|\right) t_{\mu \xi}^{\mu \nu \xi}(r) t_{\nu \xi}^{\mu \nu \xi}\left(r^{\prime}\right) .
$$

With the help of the sum rules for $c^{(3)}$, the $t(r)$ functions can be determined by solving a rather ill-conditioned integral equation. The triplet direct correlation functions are then introduced in the triplet Ornstein-Zernike equation (OZ3) to obtain the three-particle distribution function $g_{\mu \nu \xi}^{(3)}\left(\boldsymbol{r}, \boldsymbol{r}^{\prime}\right)$. The procedure to obtain $g^{(3)}$ is plagued with the numerical inaccuracies of the inverse Fourier transformation of a function which in $r$ space has the characteristic behavior of a step function.

Here, we again resorted to the GMRESNL method, although with a considerable larger number of search directions (10-20). Given the convergence difficulties we have restricted the calculations to the symmetric 3:3 electrolyte. In this case, the pair correlation functions have been discretized using a mesh in $r$ of 2048 points with a grid size of $0.04 \sigma$.

\section{RESULTS}

We present results for a strongly charged 3:3 electrolyte with varying size ratios $\lambda=\sigma_{++} / \sigma_{--}=1,2,3$ and a $1: 3$ electrolyte with $\lambda=1.707,3$, being $\sigma_{--}=2 r_{-}=2.8428 \AA$. We use reduced unit lengths defined as $r^{*}=r / \sigma_{-}$. The size ratio 1.707 roughly corresponds to $\mathrm{LaCl}_{3}$, which has been modeled previously with the primitive model. ${ }^{24}$ All cases have been studied at room temperature $298.15 \mathrm{~K}$ and the solvent medium has been modeled using the dielectric constant of water, $\epsilon=78.36$. In the 1:3 electrolyte we have also considered one case with the dielectric constant of acetonitrile, $\epsilon=36.4$, so as to illustrate the effect of lowering the potential screening on the structure. 

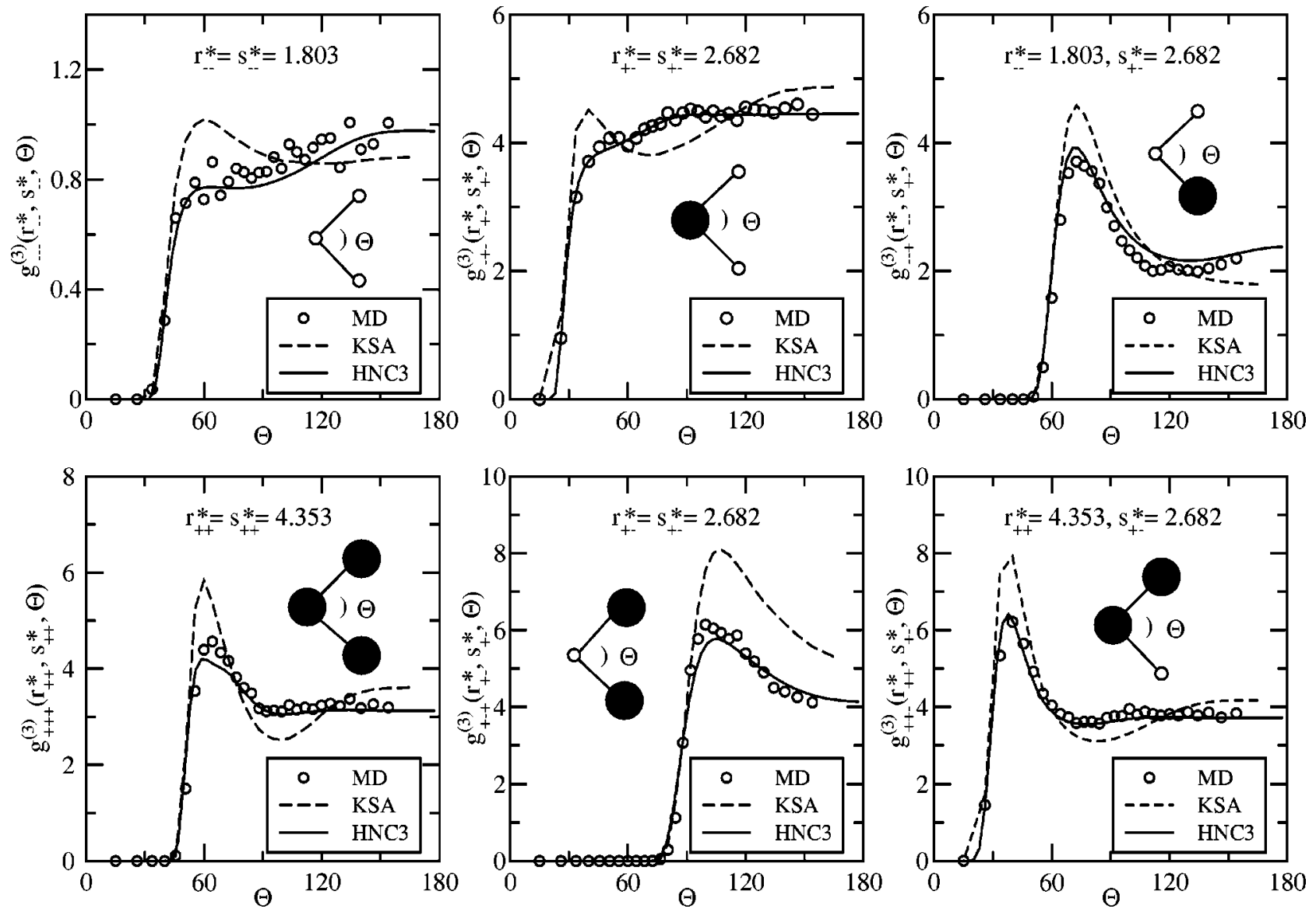

FIG. 8. Triplet distribution function for a $1: 3$ electrolyte solution with $\lambda=3.0$ at $c=0.89 \mathrm{M}$.

Reference results have been generated by means of canonical ensemble molecular dynamics (MD). ${ }^{25}$ Temperature stabilization has been carried out using a Berendsen thermostat with a time constant for the temperature coupling of 0.4 $\mathrm{ps}$, being the simulation time step $0.001 \mathrm{ps}$. Thermalization is achieved after $5 \times 10^{4}$ time steps and, depending on the case, different number of production steps were used in the calculation of average properties (see Table I). Calculations with full Coulomb interaction have been performed using Ewald's summation technique. Table I lists the states and simulation conditions considered in this work.

Now one must first assess to what extent the truncated model adequately reproduces the McMillan-Mayer electrolyte. This is illustrated in Fig. 1(a) for a 3:3 electrolyte and in Fig. 1(b) for a 1:3 system. There, the pair distribution functions are plotted for systems interacting via the truncated and shifted Coulomb (tsc) interaction and the full Coulomb potential (fcp). The case illustrated in Fig. 1(a) is rather extreme for its high ionic strength, which is apparent in the long-range ionic ordering of the fcp results. We are here dealing with a regime which starts to resemble a molten salt. Nonetheless, aside from a phase shift in the medium-long range region and the more pronounced damping of the tsc correlations, the first coordination shells are adequately accounted for by the truncated interaction. Consequently, we can expect that the picture of clustering and local order resulting from this model will be fairly adequate. In the case of the 1:3 electrolyte, which corresponds to a much weaker ionic strength, the agreement between both models is very satisfactory. Two other important quantities provide a measure of the quality of the truncated model, namely the Stillinger-Lovett zero and second moments. ${ }^{26}$ These quantities can be defined in terms of the following $r$-dependent functions:

$$
Z M_{i}(r)=-1-\left(1 / Z_{i}\right) \int_{0}^{r}\left[\sum_{j=1}^{n} Z_{j} \rho_{j} g_{i j}\left(r^{\prime}\right)\right] 4 \pi\left(r^{\prime}\right)^{2} d r^{\prime},
$$

which in the thermodynamic limit must comply with the zero moment condition, $\lim _{r \rightarrow \infty} Z M_{i}(r)=0$, and

$$
\begin{aligned}
S M(r)= & -1-\left(4 \pi e^{2} / 6 \epsilon k T\right) \\
& \times \int_{0}^{r}\left[\sum_{i=1}^{n} \sum_{j=1}^{n} Z_{i} Z_{j} \rho_{i} \rho_{j} g_{i j}\left(r^{\prime}\right)\left(r^{\prime}\right)^{2}\right] 4 \pi\left(r^{\prime}\right)^{2} d r^{\prime},
\end{aligned}
$$

which should fulfill the second moment condition $\lim _{r \rightarrow \infty} S M(r)=0$. These quantities are plotted in Fig. 2 for the tsc and fcp models for one of our case studies. For distances below $10 \sigma_{--}$the tsc model agrees very well with the fcp. Beyond the cutoff distance deviations become apparent and the tsc system does not fulfill the moment conditions.

Concerning the theoretical predictions, in Figs. 3(a) and 3(b) we present the pair distribution functions computed in the HNC and HNC3 approximations. The shortcomings of 

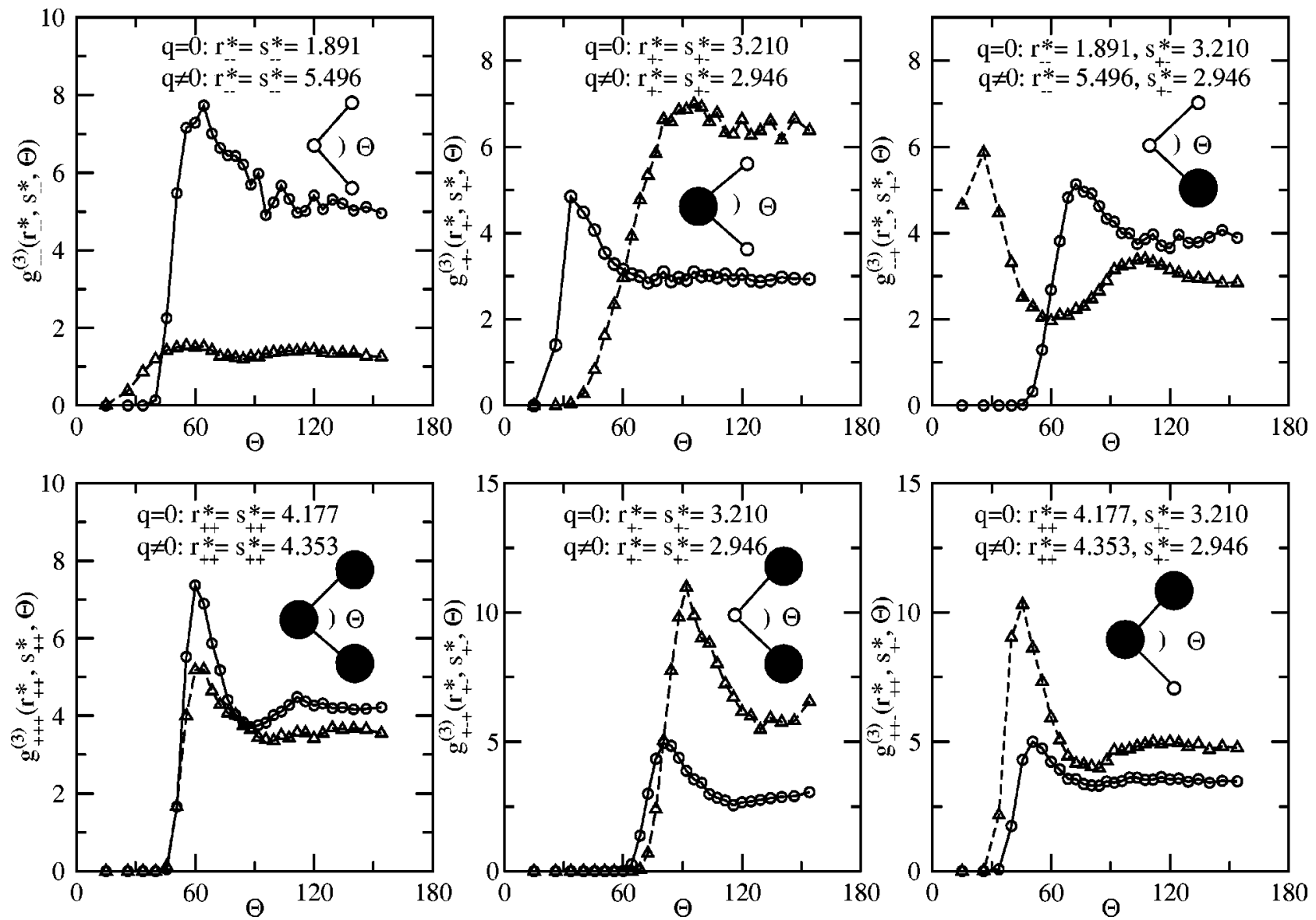

FIG. 9. Triplet distribution function for a 3:3 soft sphere mixture with $\lambda=3.0$ and packing fraction $\eta=0.1806$ (equivalent $c=0.89 \mathrm{M}$ ) vs the corresponding charged 3:3 electrolyte. Circles: uncharged system. Triangles: charged system. Lines have also been depicted as a guide.

the HNC equation are apparent especially in the first maximum of the like distribution functions (in the 1:3 case for $g_{++}$, which corresponds to the largest particles). This peak is due to the presence of clusters of particles with alternating charge signs $(+-+$ or -+-$)$, i.e., one should go beyond the pairing level to get a correct description. The improvement of the HNC3 over the HNC is notorious in both cases, and this is in accordance with previous findings for simple fluids ${ }^{10}$ and mixtures. ${ }^{11}$ Similar results are also found for other cases studied.

As to the triplet structure, in Fig. 4 we present the $g^{(3)}\left(r, s, \theta_{12}\right)$ functions for a 3:3 symmetric electrolyte comparing high and low concentration results. The values of $r$ and $s$ have been chosen to correspond to the position of the relevant peaks of the pair distribution functions. The fact that the BHP approximation yields nonzero $g^{(3)}$ for low angleswhich for the given configuration corresponds to overlapping particles-stems from numerical inaccuracies inherent to the multidimensional Fourier inversion ${ }^{11}$ of the $\mathrm{OZ} 3$ equation. Aside from this deficiency this approximation qualitatively reproduces the behavior of the like triplet distribution function in agreement with the $\mathrm{HNC} 3$ and in marked contrast with the Kirkwood superposition approximation (KSA). We note in passing that some of the deficiencies exhibited by the HNC3 come from inaccuracies in the determination of the height of the pair distribution peaks which enter into the triplet function via Eq. (8). This is particularly noticeable in the wide angle (i.e., large $r$ ) region.

As the concentration is increased, the $g_{---}^{(3)}=g_{+++}^{(3)}$ distribution functions develop an additional maximum situated around $90^{\circ}$. Although the KSA captures this feature, it clearly misses its importance reversing the relative heights of the peaks. In $g_{-+-}^{(3)}$ it is shown that the maximum at $85^{\circ}$ spreads out as density is decreased and turns into a plateau that reaches out $180^{\circ}$, i.e., fully linear structures. This is the signature of the presence of open clusters in the low density regime. Finally, in $g_{--+}^{(3)}$ one observes that at low concentrations the +- attraction places the maximum at low angles whereas the third particle becomes noticeable at high concentrations inducing a structure that resembles a pair distribution function, with a first peak appearing at distances determined by the core repulsion of the third particle. This effect however is also accounted for by the KSA.

When the cation size is doubled, the symmetry in the +-+ and -+- triplet functions breaks (see Fig. 5) and both functions are less structured than their symmetric counterpart. It is noticeable that $g_{+-+}^{(3)}$ exhibits a maximum around $100^{\circ}$ while $g_{-+-}^{(3)}$ does not. Notice that, although concentration has not changed, the volume occupied by the cations has increased by a factor $\lambda^{3}$ with respect to the symmetric case-compare the packing fractions $\eta=\pi\left(\rho_{-} \sigma_{--}^{3}\right.$ 
$\left.+\rho_{+} \sigma_{++}^{3}\right) / 6$ in Table I. Thus, packing effects start to become apparent and the result is that clusters with open configurations (high angles) are less favored simply because they are not compatible with a situation that places more ions somewhere in their neighborhood. This effect is more pronounced for the larger ions than for the smaller ones which may move more freely among the big ones. In this way, the cations are more correlated than the ions at intermediate angles, and hence the prevalence of the maximum in $g_{+-+}^{(3)}$ with respect to $g_{-+-}^{(3)}$. In fact, if the size asymmetry is further increased $(\lambda=3)$, packing effects force anions to remain in the cavities between cations and the peak in $g_{+-+}^{(3)}$ grows (results not shown). This is partially illustrated in Figs. 6 and 7 where simulation snapshots for a 3:3 electrolyte for $\lambda=1$ and $\lambda$ $=3$ are presented. In the first case clustering effects are readily seen, i.e., particles are not uniformly distributed with empty regions in the simulation box. Also most ions tend to be surrounded by particles of opposite charge and a majority of particle arrangements correspond to relatively open structures $\left(\theta_{12} \gtrsim 100^{\circ}\right)$. In Fig. 7, on the contrary, cations almost uniformly fill the volume and anions move within the remaining available space. If one is to observe the same type of association in this system as was found in Fig. 6, it would be necessary to decrease the concentration to levels where the convergence of the HNC3 breaks down.

In Fig. 8 we present results for the fully asymmetric system, i.e., a 1:3 electrolyte with $\lambda=3.0$, where the excellent performance of the HNC3 is clearly apparent. In this case, the KSA fails to reproduce the qualitative behavior of various like and unlike triplet distribution functions. The qualitative trends found in Fig. 5 are again encountered here, enhanced by the larger size ratio. Once more the competing factors are packing effects and Coulomb interactions. To illustrate how the charge alters the triplet structure of the fluid even in cases where packing effects are of leading importance, in Fig. 9 we present simulation results obtained for an uncharged equimolar soft sphere mixture (plain Ramanathan-Friedman core repulsions) corresponding to a system with $\lambda=3$ and $\eta=0.1806$, and compare them with those of the corresponding 3:3 charged system. One first observes the huge decrease in $g_{--}^{(3)}(--$ now denotes the anions and the small particles) due to the charge repulsions which dominate the behavior of the small particles. This repulsion is also the source of the disappearance of the peak at $30^{\circ}$ present in $g_{-+-}^{(3)}$ in the uncharged system and the shift to low angles in the maximum of $g_{--+}^{(3)}$. On the other hand, the arrangement of large particles is mostly conditioned by steric effects. Summarizing, as one should expect the charge affects the reorganization of the small particles in a "matrix" of large particles which conserves its relative local ordering despite the presence of charges. This is also seen in the $g_{++}^{(2)}$ (not illustrated) whose shape remains unaltered by the charges although the maxima are located at larger separations to accommodate to the charge repulsion.

Finally in Fig. 10 we analyze the effect of lowering the dielectric constant (i.e., the screening) in the 1:3 electrolyte that aims at modeling $\mathrm{LaCl}_{3}$. One observes a considerable increase in the height of the peaks denoting a larger association. Also the shift to lower angles in $g_{--+}^{(3)}$ results from the

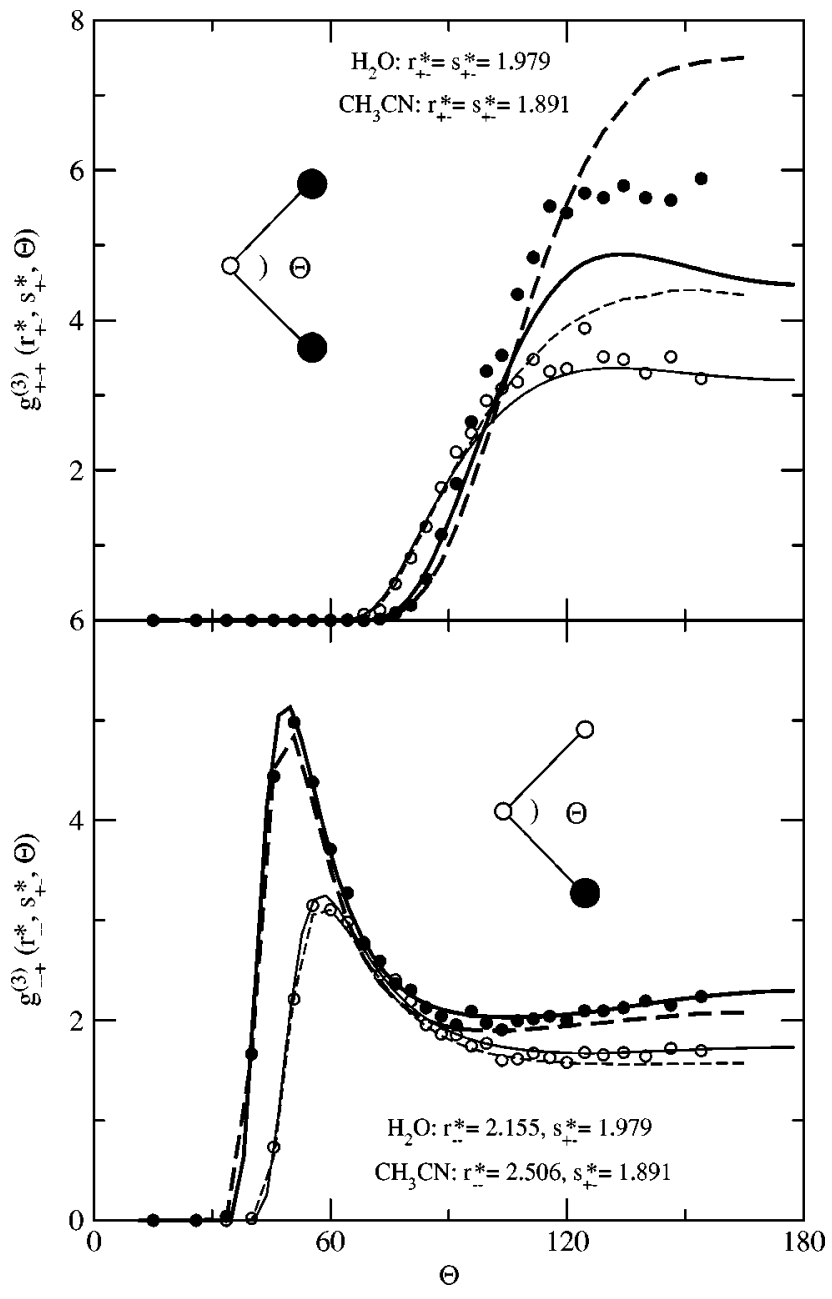

FIG. 10. Influence of the dielectric constant on the triplet distribution function for a 1:3 electrolyte solution with $\lambda=1.707$ at $c=1.33 \mathrm{M}$. Open symbols are MD results for $\epsilon=78.36$ and closed symbols correspond to $\epsilon$ $=36.4$. KSA data are represented by dashed curves and HNC3 by solid lines. Thin lines correspond to $\epsilon=78.36$ and thick ones to $\epsilon=36.4$.

increase in the attraction between the -+ particles. Once more $g_{+-+}^{(3)}$ is more dominated by steric effects and the performance of KSA is poorer than for $g_{--+}^{(3)}$. Again the mismatch in the $\mathrm{HNC} 3$ results for large angles results from an underestimation of the heights of the pair distribution function.

In summary, we have presented a detailed analysis of the performance of the HNC3 results to analyze the local structure of symmetric and asymmetric electrolytes at the level of pair and triplet structure. In general the HNC3 approximation performs satisfactorily for the cases studied. One could thus rely on this approximation to provide estimates for the triplet distribution functions in charged systems. A further development should provide an efficient and accurate route to calculate the triplet direct correlation function from the quantities resulting from the HNC3 formalism. This latter function is a key ingredient in the study of phenomena such as the freezing ${ }^{27}$ or even the dynamics of supercooled systems. ${ }^{28}$ Work in this direction is planned.

\section{ACKNOWLEDGMENTS}

This work was funded by the Spanish Dirección General de Enseñanza Superior e Investigación Científica under 
Grant No. BFM2001-1017-C03. S.J. acknowledges financial support from the Universidad Complutense de Madrid.

${ }^{1}$ W. J. McMillan and J. E. Mayer, J. Chem. Phys. 13, 276 (1945).

${ }^{2}$ G. M. Abernethy and M. J. Gillan, Mol. Phys. 39, 839 (1980).

${ }^{3}$ F. Lado, S. M. Foiles, and N. W. Ashcroft, Phys. Rev. A 28, 2374 (1983).

${ }^{4}$ J. P. Hansen and I. R. McDonald, Theory of Simple Liquids (Oxford University Press, Oxford, 1986).

${ }^{5}$ M. J. Gillan, Mol. Phys. 38, 1781 (1979).

${ }^{6}$ D.-M. Duh and A. D. J. Haymet, J. Chem. Phys. 100, 2244 (1994).

${ }^{7}$ F. Bresme, E. Lomba, J. J. Weis, and J. L. F. Abascal, Phys. Rev. E 51, 289 (1995).

${ }^{8}$ M. S. Wertheim, J. Chem. Phys. 85, 2929 (1986).

${ }^{9}$ Y. V. Kalyuzhnyi and G. Stell, Mol. Phys. 78, 1247 (1993); Y. V. Kalyuzhnyi, V. Vlachy, M. F. Holovko, and G. Stell, J. Chem. Phys. 102, 5770 (1995).

${ }^{10}$ P. Attard, J. Chem. Phys. 91, 3072 (1989).

${ }^{11}$ S. Jorge, E. Lomba, and J. L. F. Abascal, J. Chem. Phys. 114, 3562 (2001).

${ }^{12}$ R. Kjellander and S. Sarman, Mol. Phys. 70, 215 (1990).

${ }^{13}$ D. G. Triezenberg and R. Zwanzig, Phys. Rev. Lett. 28, 1183 (1972).

${ }^{14}$ E. Lomba, S. Jorge, and M. Alvarez, Phys. Rev. E 63, 011203 (2001)

${ }^{15}$ J. L. Barrat, J. P. Hansen, and G. Pastore, Mol. Phys. 63, 747 (1988).
${ }^{16}$ S. Jorge, G. Kahl, E. Lomba, and J. L. F. Abascal, J. Chem. Phys. 113, 3302 (2000)

${ }^{17}$ A. R. Allnat, Mol. Phys. 8, 533 (1964).

${ }^{18}$ P. S. Ramanathan and H. L. Friedman, J. Chem. Phys. 54, 1086 (1970).

${ }^{19}$ P. Linse and H. C. Andersen, J. Chem. Phys. 85, 3027 (1986).

${ }^{20}$ J. C. G. Montoro, F. Bresme, and J. L. F. Abascal, J. Chem. Phys. 101, 10892 (1994).

${ }^{21}$ P. J. Rossky, J. B. Dudowicz, B. L. Tembe, and H. L. Friedman, J. Chem. Phys. 73, 3372 (1980).

${ }^{22}$ C. L. Brooks III, B. M. Pettitt, and M. Karplus, J. Chem. Phys. 83, 5897 (1985)

${ }^{23}$ Y. Saad and M. H. Schultz, SIAM (Soc. Ind. Appl. Math.) J. Sci. Stat. Comput. 7, 856 (1986); Tech. Report No. 254, Yale University, 1993.

${ }^{24}$ J.-P. Simonin, O. Bernard, and L. Blum, J. Phys. Chem. B 102, 4411 (1998); B. Hribar, V. Vlachy, and O. Pizio, ibid. 104, 4479 (2000).

${ }^{25}$ DL_POLY is a package of molecular simulation routines written by $\mathrm{W}$. Smith and T. R. Forester. Copyright: The Engineering and Physical Sciences Research Council, acting through its Daresbury and Rutherford Appleton Laboratory at Daresbury Laboratory, 1994.

${ }^{26}$ F. H. Stillinger and R. Lovett, J. Chem. Phys. 49, 1991 (1968).

${ }^{27}$ A. R. Denton and N. W. Ashcroft, Phys. Rev. A 43, 3161 (1991).

${ }^{28}$ F. Sciortino and W. Kob, Phys. Rev. Lett. 86, 648 (2001). 